Research Paper

\title{
Efficacy and safety of nimotuzumab in addition to radiotherapy and temozolomide for cerebral glioblastoma: a phase II multicenter clinical trial
}

\author{
Xiao-Jing Du' ${ }^{1}$, Xian-Ming $\mathrm{Li}^{2}$, Lin-Bo Cai ${ }^{3}$, Jian-Cong Sun ${ }^{4}$, Si-Yang Wang, ${ }^{5}$ Xi-Cheng Wang6, Xiao-Lin \\ Pang1, Mei-Ling Deng ${ }^{1}$, Fang-Fang Chen ${ }^{1}$, Zhi-Qiang Wang ${ }^{1}$, Fu-Rong Chen ${ }^{7}$, Hong-Hong Zhang1, \\ Hui-Yun Wang 7 , Patricia Piedra ${ }^{8}$, Zhong-Ping Chen ${ }^{9}$, Jun Lin ${ }^{10}$, Shao-Xiong Wu ${ }^{1 凶}$
}

1. Department of Radiation Oncology, Sun Yat-sen University Cancer Center; State Key Laboratory of Oncology in South China; Collaborative Innovation Center for Cancer Medicine, No. 651 Dongfeng Road East, Guangzhou 510060, China

2. Department of Radiation Oncology, Shenzhen People's Hospital, No. 1017 Dongmen Road North, Luohu District, Shenzhen 518020, China

3. Department of Radiation Oncology, Guangdong 999 Brain Hospital, No. 578 Shatai Road South, Guangzhou 510510, China

4. Department of Radiation Oncology, The First Affiliated Hospital of Guangzhou Medical University, No. 151 Yanjiang Road West, Guangzhou 510120, China

5. Department of Radiation Oncology, The 5th Affiliated Hospital of Sun Yat-sen University, No. 52 Meihua Road East, Zhuhai, 519000, China

6. Department of Radiation Oncology, The First Affiliated Hospital/School of Clinical Medicine of Guangdong Pharmaceutical University, No. 19 Nonglin Xia Road, Guangzhou 510080, China

7. Sun Yat-sen University Cancer Center; State Key Laboratory of Oncology in South China; Collaborative Innovation Center for Cancer Medicine, No. 651 Dongfeng Road East, Guangzhou 510060, China

8. Center of Molecular Immunology, Avenue 15 and 216 St., Siboney, Playa, La Habana, Cuba. A.P 16040, La Habana 11600, Cuba

9. Department of Neuro-Oncology, Sun Yat-sen University Cancer Center; State Key Laboratory of Oncology in South China; Collaborative Innovation Center for Cancer Medicine, No. 651 Dongfeng Road East, Guangzhou 510060, China

10. Department of Anesthesiology, Stony Brook University, School of Medicine, Health Sciences Tower, Level 4, Rm 060, Stony Brook, NY 11794-8480, United States

$\square$ Corresponding author: Shao-Xiong Wu, M.D., Department of Radiation Oncology, Sun Yat-sen University Cancer Center; State Key Laboratory of Oncology in South China; Collaborative Innovation Center for Cancer Medicine, No. 651 Dongfeng Road East, Guangzhou 510060, China. Telephone: +86-20-87343384; Fax: +86-20-87343384; E-mail: wushx@sysucc.org.cn

( ) Ivyspring International Publisher. This is an open access article distributed under the terms of the Creative Commons Attribution (CC BY-NC) license (https:// creativecommons.org/licenses/by-nc/4.0/). See http://ivyspring.com/terms for full terms and conditions.

Received: 2018.09.22; Accepted: 2019.04.13; Published: 2019.06.02

\begin{abstract}
Background: Nimotuzumab is a humanized anti-epidermal growth factor receptor (EGFR) antibody that has shown preclinical and clinical anticancer activity in cerebral glioblastoma multiforme (GBM). We conducted a phase II, single-arm, multicenter clinical trial to evaluate the benefit of adding nimotuzumab to current standard chemo-radiotherapy for patients with GBM with positive EGFR expression.

Methods: Newly diagnosed patients with histologically proven single supratentorial GBM and epidermal growth factor receptor (EGFR) positive expressions were recruited. All patients were treated with nimotuzumab, administered once a week intravenously for 6 weeks in addition to radiotherapy with concomitant and adjuvant temozolomide after surgery. The primary endpoints were overall survival (OS) and progression-free survival (PFS). Secondary objectives included objective response rate (ORR) and toxicity.

Results: A total of 39 patients were enrolled and 36 patients were evaluated for efficacy. The ORR at the end of RT was $72.2 \%$. Median OS and PFS were 24.5 and 11.9 months. The 1 -year OS and PFS rates were 83.3\% and $49.3 \%$. The 2-year OS and PFS rates were $51.1 \%$ and $29.0 \%$. $O$ (6)-methylquanine DNA methyl-tranferase (MGMT) expression is known to affect the efficacy of chemotherapy and status of its expression is examined. No significant correlation between treatment outcomes and MGMT status was found. Most frequent treatment-related toxicities were mild to moderate and included constipation, anorexia, fatigue, nausea, vomiting, and leucopenia.

Conclusions: Our study show that nimotuzumab in addition to standard treatment is well tolerable and has increased survival in newly diagnosed GBM patients with EGFR positive expression.
\end{abstract}

Key words: glioblastoma; radiotherapy; temozolomide; nimotuzumab; epidermal growth factor receptor; $\mathrm{O}$ (6)-methylquanine DNA methyl-tranferase (MGMT) 


\section{Background}

Glioblastoma multiforme (GBM) is the most common and aggressive primary brain tumor in adults, accounting for approximately $60-70 \%$ of gliomas [1]. Due to the resistance of GBM cells to treatment and its invasive nature, GBM patients have poor outcomes, with less than $5 \%$ of patients surviving up to 5 years after diagnosis [2]. The current standard therapy for newly diagnosed GBM includes maximal safe resection followed by radiotherapy (RT) and concomitant temozolomide (TMZ) chemotherapy and up to 6 months of adjuvant TMZ (TMZ/RT $\rightarrow$ TMZ). However, this strategy only successfully prolonged the median survival time to 14.6 months [3]. Most GBM patients still dies within 2 years, necessitating exploration of other treatment options.

The epidermal growth factor receptor (EGFR), a tyrosine kinase receptor, was discovered as a proto-oncogene [4]. The overexpression of EGFR and its ligands in several carcinomas and their association with aggressive tumor development provides a rationale for selectively targeting this signal pathway. EGFR is also one of the most promising treatment targets for patients with GBM after the demonstration of gene amplification and protein overexpression in some GBM tumors [5-7]. It has been reported that about half of those tumors overexpression is the result of a mutant form of the receptor called as EGFR variant III mutant (EGFRvIII). EGFRvIII has a kinase domain that leads to constitutive signaling pathway activation, further enhance tumorigenic activity, promote tumor progression and drug resistance [8,9].

Nimotuzumab is a humanized anti-EGFR antibody, which has been extensively evaluated and granted approval for use in patients with head and neck squamous carcinoma $[10,11]$. Recently, several clinical trials have confirmed the clinical activity of nimotuzumab in treating adult high-grade glioma patients [12-17]. However, the effect of adding nimotuzumab to current standard TMZ/RT $\rightarrow \mathrm{TMZ}$ is still controversial. Thus, this study aimed to investigate the clinical benefit and safety of adding nimotuzumab to the standard treatment for patients with newly diagnosed GBM. Since O (6)-methylquanine DNA methyl-tranferase (MGMT) expression affects efficacy of TMZ treatment, we also investigate the effect of adding nimotuzumab to current standard TMZ/RT $\rightarrow \mathrm{TMZ}$ in MGMT expression patients.

\section{Patients and Methods}

\section{Study Design}

This multicenter, single-arm, phase II trial investigated the efficacy and safety of nimotuzumab added to TMZ/RT $\rightarrow \mathrm{TMZ}$ in patients with newly diagnosed GBM (ClinicalTrials.gov identifier NCT03388372). The study was approved by the Institutional Review Board of Sun Yat-sen University Cancer Center, and was conducted in accordance with the Good Clinical Practice guideline. The ethics approval was for all the study sites. The investigator obtained signed informed consent form from patients for approval of treatment and for personal information use in research without violating their privacy. The primary endpoints were the overall survival (OS) and progression-free survival (PFS). Secondary endpoints were objective response rate (ORR) and safety. The influence of MGMT expression status on efficacy was investigated in a prospectively planned subgroup analysis.

\section{Patients}

Main inclusion criteria were age between 18 - 75 years; newly diagnosed, histologically proven single supratentorial GBM (WHO grade 4); EGFR positive; $>50 \%$ of the gross tumor volume removed by surgery; Karnofsky performance score (KPS) $\geq 60$; and adequate renal function (creatinine $\leq 1.5 \times$ upper limit of normal [ULN] or creatinine clearance $\geq 60$ $\mathrm{mL} / \mathrm{min}$ ), hepatic function (total bilirubin $\leq 1.5 \times \mathrm{ULN}$ and serum transaminases $\leq 3 \times U L N)$, and hematologic function (white blood cell count $\geq$ $3,000 / \mathrm{uL}$ or absolute neutrophil count $\geq 1,500 / \mathrm{uL}$, platelets $\geq 100,000 / \mathrm{uL}$, and hemoglobin $\geq 10 \mathrm{~g} / \mathrm{dL}$ ). Tumor tissue was required for central pathology review and re-checking EGFR and MGMT expression status. An interval of 2 to 6 weeks between surgery and RT was required.

The exclusion criteria were negative EGFR expression, prior chemotherapy, anti-EGFR therapy, RT, or a history of malignancy in the previous 5 years. Patients with severe complications or active infection, continuous vomiting that could interfere with the oral administration of TMZ were also excluded. A negative pregnancy test and adequate contraception were mandatory.

\section{Treatment}

All patients had received surgery under the principle of maximum safe resection of tumors with at least $50 \%$ of the gross tumor volume removed. Fractionated three-dimensional conformal RT was given at 2.0 Gy per fraction with five daily fractions per week for 6 weeks. Gross tumor volume (GTV) delineation was based on the resection cavity plus any residual enhancing tumor on contrast-enhanced T1 weighted magnetic resonance imaging (MRI) and area of hyperintensity on FLAIR/T2 when suspicious for tumor rather than edema. The clinical target volume-1 
(CTV1) was defined as GTV with a $2 \mathrm{~cm}$ margin and CTV2 was defined as GTV with a $1 \mathrm{~cm}$ margin. CTVs with $0.3 \mathrm{~cm}$ margins were defined as planning tumor volumes (PTVs). The prescription doses were 50 Gy to PTV1 and 60 Gy to PTV2. TMZ at $75 \mathrm{mg} / \mathrm{m}^{2}$ daily was administered orally from the first to the last day of RT. After a 4-week break, individualized adjuvant TMZ regimen was given based on MGMT expression status. The standard 5-day schedule every 4 weeks for six cycles was given for patients with negative MGMT expression. The dose was $150 \mathrm{mg} / \mathrm{m}^{2}$ for the first cycle and $200 \mathrm{mg} / \mathrm{m}^{2}$ starting from the second cycle. The 7-day on/7-day off schedule every 2 weeks for twelve cycles was given for patients with positive MGMT expression. The dose was $100 \mathrm{mg} / \mathrm{m}^{2}$ for the first two cycles and $150 \mathrm{mg} / \mathrm{m}^{2}$ starting from the third cycle.

Nimotuzumab of $200 \mathrm{mg}$ was administered as an over 1-hour intravenous infusion once weekly, starting from the first week and continuing until the last week of RT for a total of 6 times.

\section{EGFR and MGMT Status}

EGFR and MGMT expression were determined by immunohistochemistry analysis, using commercially available detection kits (Ascend Biotechnology, China) and automated staining techniques (Benchmark XT, Ventana, and Autostainer Link 48, Dako). EGFR positive expression was defined as $>10 \%$ of cells stained positive. Two pathologists independently interpreted the results without any clinical or other histological information.

\section{Efficacy and Safety Assessments}

MRI scan was performed both before surgery and within $24-72$ hours after surgery to identify residue ratio. MRI was also performed within 2 weeks before RT to formulate RT plan, repeated at the end of RT and every 3 months thereafter to evaluate clinical efficacy. Treatment responses were evaluated according to RANO's criteria [18]. The tumor progression was defined by the occurrence of any of the following: the sum of the products of perpendicular diameters of target enhancing lesions is increased by $\geq 20 \%$ for patients receiving stable or increasing doses of corticosteroids; a significant increase in T2/FLAIR nonenhancing lesions for patients receiving stable or increasing doses of corticosteroids; the appearance of any new lesions; clear progression of unmeasurable lesions; or definite clinical deterioration not attributable to other causes apart from the tumor. When there was tumor progression, patients were treated at the investigator's discretion, and the regimen of second-line therapy was recorded.

Safety was evaluated by adverse events (AEs), laboratory assessments, and physical examinations, including KPS, neurologic examinations, weekly blood counts, serum chemistry, urinalysis, chest x-ray, and ECG. All AEs were recorded according to Common Toxicity Criteria version 3.0, and the AEs possibly related to treatment with TMZ/RT or nimotuzumab or not otherwise attributed to other causes were reported as treatment-related AEs. AEs were reported separately for the RT period and the adjuvant-therapy period. The former was defined as from day 1 of RT to 28 days after the last day of RT, or to the first day of adjuvant TMZ therapy. The latter was defined as from the first day of adjuvant TMZ therapy to the 35 th days since day 1 of the last cycle of TMZ

\section{Statistical analysis}

This study used Simon's optimal two-stage design, which was formulated to distinguish a favorable one-year OS rate of $87 \%$ from a control rate of $66 \%$ with $90 \%$ power $[3,19]$, at a significance level of 0.05 . In total, 35 patients need to be enrolled.

The analyses were performed on the per-protocol population, defined as patients who received at least four cycles of nimotuzumab and at least 4 weeks of concomitant TMZ. PFS was defined as time from surgery to progression or death from any cause. OS was defined as time from surgery to death from any cause. All calculations were performed using Statistical Package for the Social Sciences, version 20.0 (SPSS, Chicago, IL, USA). Survival curves were made with the Kaplan-Meier method and compared with the log-rank test. Cox proportional hazards model was applied to adjust for stratification factors and other confounding variables. Two-tailed P-values $<0.05$ were considered significant.

\section{Results}

\section{Patient characteristics}

From August 2010 to July 2015, 39 patients from 6 institutions were recruited, 3 patients with confirmed protocol deviation were eliminated from the analysis (one started RT after a delay of 19.3 weeks after surgery; one received other anti-tumor therapy after RT; one has not completed RT due to personal reason). Among the 36 per-protocol patients, 20 (55.6\%) had positive MGMT expression. EGFR immunohistochemistry showed strong positive staining on cell membrane of all the patients (Figure 1), which was in consistent with the result reported by Yoon et al [20]. The patient characteristics are shown in Table 1.

\section{Treatment Delivery}

All 36 patients completed the treatment with nimotuzumab plus concomitant TMZ/RT. Among 
them, $32(88.9 \%)$ patients completed at least one cycle of adjuvant TMZ and $4(11.1 \%)$ didn't enter the adjuvant-therapy period because of tumor progression. Twenty-four $(66.7 \%)$ patients completed all 6 planned cycles of adjuvant TMZ. One (2.8\%) patient completed 5 cycles, 2 (5.6\%) patients completed 3 cycles, one $(2.8 \%)$ patient completed 2 cycles and $4(11.1 \%)$ patients completed one cycle. Reasons for early discontinuation of adjuvant TMZ (1 - 5 cycles) were tumor progression (4 patients; $11.1 \%$ ), AEs (2 patients; $5.6 \%)$, or other personal reasons (2 patient; $5.6 \%)$.

Table 1. Baseline patient characteristics $(N=36)$

\begin{tabular}{|c|c|c|}
\hline Variable & No. & $\%$ \\
\hline \multicolumn{3}{|l|}{ Age, years } \\
\hline Median age (range) & $49(18-69)$ & \\
\hline$\leq 40$ & 8 & 22.2 \\
\hline$>40$ & 28 & 77.8 \\
\hline \multicolumn{3}{|l|}{ Sex } \\
\hline Male & 19 & 52.8 \\
\hline Female & 17 & 47.2 \\
\hline \multicolumn{3}{|c|}{ Karnofsky performance score } \\
\hline $90-100$ & 25 & 69.4 \\
\hline $60-80$ & 11 & 30.6 \\
\hline \multicolumn{3}{|l|}{ Tumor location } \\
\hline \multicolumn{3}{|l|}{ Hemisphere } \\
\hline Left & 22 & 61.1 \\
\hline Right & 14 & 38.9 \\
\hline \multicolumn{3}{|l|}{ Lobe } \\
\hline Frontal lobe & 11 & 30.6 \\
\hline Others & 25 & 69.4 \\
\hline \multicolumn{3}{|c|}{$\begin{array}{l}\text { O6-methylguanine-DNA methyltransferase } \\
\text { expression }\end{array}$} \\
\hline Negative & 16 & 44.4 \\
\hline Positive & 20 & 55.6 \\
\hline \multicolumn{3}{|c|}{$\begin{array}{l}\text { Response of combination therapy at the end of } \\
\text { radiotherapy }\end{array}$} \\
\hline Complete Remission & 20 & 55.6 \\
\hline Partial Remission & 6 & 16.7 \\
\hline Stable Disease & 6 & 16.7 \\
\hline Progressive Disease & 4 & 11.1 \\
\hline \multicolumn{3}{|l|}{ Surgery } \\
\hline Total resection & 15 & 41.7 \\
\hline Sub-total resection & 21 & 58.3 \\
\hline
\end{tabular}

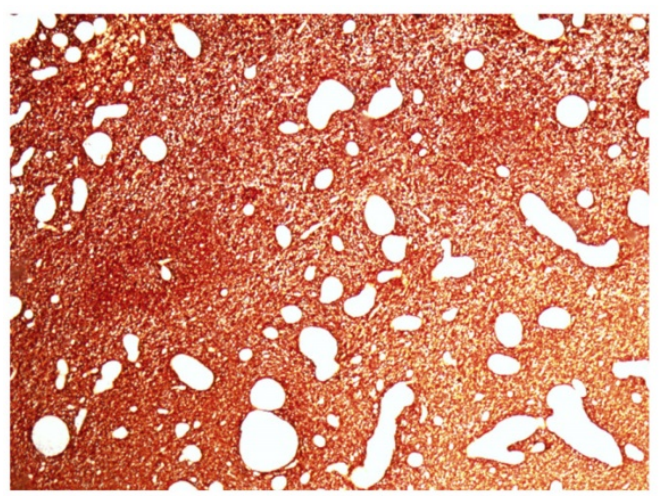

A

\section{Efficacy}

At the end of RT, 26 (72.2\%) patients had objective response, including $20(55.6 \%)$ for complete remissions (CR) and $6(16.7 \%)$ for partial remissions (PR). Six (16.7\%) patients had stable disease (SD). $4(11.1 \%)$ patients had progressive disease (PD).

March 23rd, 2017 was the end of follow-up. The median follow-up was 23.4 months (range 8.3-66.0). During follow-up, 27 (75.0\%) patients progressed and $25(69.4 \%)$ patients died. The estimated OS rate was $83.3 \%$ (95\% CI 71.1-95.5) at first year, and 51.1\% (95\% CI 34.4-67.8) at second year. The median OS was 24.5 months (95\% CI 15.7-33.3) (Table 2, Figure 2). The estimated PFS rate was $49.3 \%$ (95\% CI 32.8-65.8) at first year, and $29.0 \%$ (95\% CI 13.9-44.1) at 2nd year. The median PFS was 11.9 months (95\% CI 5.5-18.2) (Table 2, Figure 3). There were similar one-year OS rates $(80.0 \%$ vs. $87.5 \%)$, and two-year OS rates $(53.3 \%$ vs. $47.7 \%)$, one-year PFS rates $(49.1 \%$ vs. $50.0 \%)$, two-year PFS rates $(27.3 \%$ vs. $31.3 \%)$ between the patients with positive or negative MGMT expression. There is no significant difference in the median OS (24.5 vs. 22.9 months, $P=0.527$, Figure 1 ) and the median PFS (9.1 vs. 11.9 months, $P=0.752$, Figure 2) between the two groups.

Univariate analysis for known clinical prognostic factors demonstrated prolonged PFS and OS in patients younger than 40 years of age $(P=0.016$ and 0.018 , respectively). $\mathrm{CR}$ at the end of RT was confirmed as a prognostic factor for better PFS $(P=$ 0.011). Besides, both CR at the end of RT and high KPS (90-100) seemed to be favorable factors for OS with marginally significance $(P=0.075$ and 0.080 , respectively, Table 3).

Multivariate analysis for PFS and OS was performed to adjust for all the factors listed in Table 3. Only older age ( $>40$ years) was confirmed as an independent predictive factor for poor PFS and OS $(P$ $=0.016$ and 0.018 , respectively).

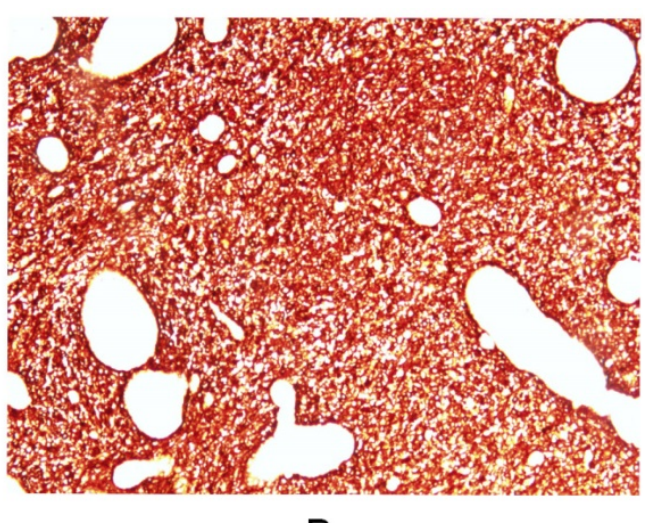

B

Figure 1. EGFR immunohistochemistry revealed strong positivity on cell membrane of glioblastomas. Magnification $\times 40(\mathrm{~A})$ and $\times 100(\mathrm{~B})$. Similar immunoreactivities are noted in all of the patients enrolled. 
A

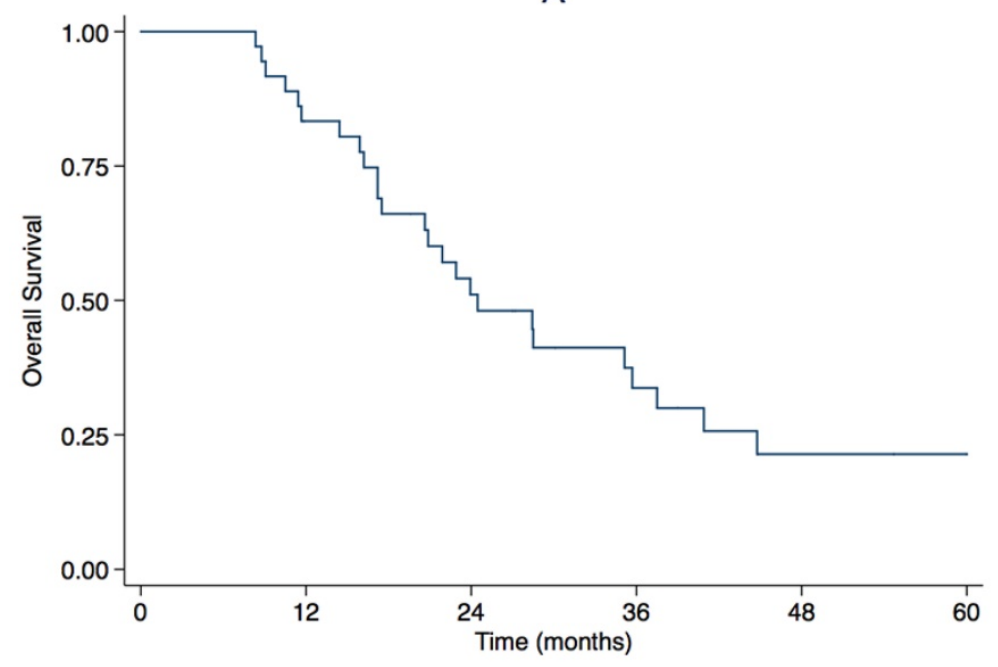

B

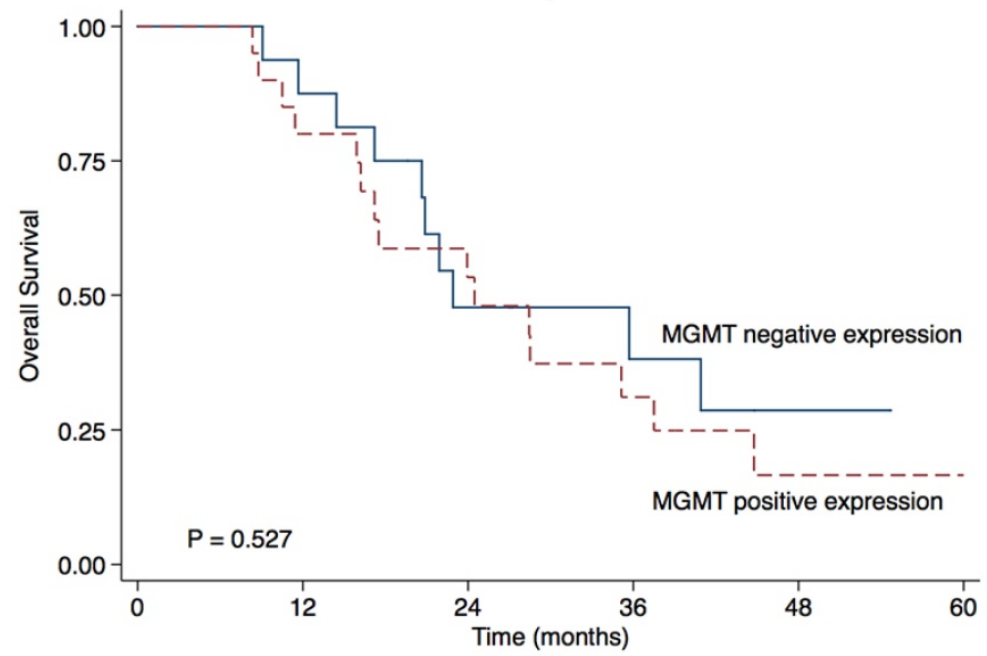

Figure 2. Kaplan-Meier estimate for overall survival for (A) all patients and stratified by (B) O6-methylguanine-DNA methyltransferase (MGMT) promoter methylation status.

Table 2. Efficacy analysis

\begin{tabular}{|c|c|c|c|c|c|c|c|c|c|}
\hline \multirow[t]{2}{*}{ Outcome } & \multicolumn{3}{|c|}{$\begin{array}{l}\text { PP population } \\
(n=36)\end{array}$} & \multicolumn{3}{|c|}{$\begin{array}{l}\text { Patients with positive } \\
\text { MGMT expression } \\
(n=20)\end{array}$} & \multicolumn{3}{|c|}{$\begin{array}{l}\text { Patients with negative } \\
\text { MGMT expression } \\
(n=16)\end{array}$} \\
\hline & No. & $\%$ & $95 \% \mathrm{CI}$ & No. & $\%$ & $95 \% \mathrm{CI}$ & No. & $\%$ & $95 \% \mathrm{CI}$ \\
\hline \multicolumn{10}{|l|}{ Response $^{*}$} \\
\hline Yes & 26 & 72.2 & & & & & & & \\
\hline No & 10 & 27.8 & & & & & & & \\
\hline \multicolumn{10}{|l|}{$\begin{array}{l}\text { Type of } \\
\text { response }\end{array}$} \\
\hline $\mathrm{CR}$ & 20 & 55.6 & & 12 & 60.0 & & 9 & 56.3 & \\
\hline PR & 6 & 16.7 & & 3 & 15.0 & & 3 & 18.8 & \\
\hline SD & 6 & 16.7 & & 1 & 5.0 & & 4 & 25.0 & \\
\hline PD & 4 & 11.1 & & 4 & 20.0 & & 0 & & \\
\hline $\begin{array}{l}\text { Median } \\
\text { PFS (mos) }\end{array}$ & 11.9 & & $5.5-18.2$ & 9.1 & & $0-21.7$ & 11.9 & & $10.5-13.2$ \\
\hline \multicolumn{10}{|l|}{ PFS rate } \\
\hline $6 \mathrm{mos}$ & & 83.3 & $71.1-95.5$ & & 75.0 & $56.0-94.0$ & & 93.8 & $81.8-100$ \\
\hline $12 \mathrm{mos}$ & & 49.3 & $32.8-65.8$ & & 49.1 & $26.8-71.4$ & & 50.0 & $25.5-74.5$ \\
\hline $18 \mathrm{mos}$ & & 34.8 & $18.9-50.7$ & & 38.2 & $16.2-60.2$ & & 31.3 & $8.6-54.0$ \\
\hline $24 \mathrm{mos}$ & & 29.0 & $13.9-44.1$ & & 27.3 & $7.1-47.5$ & & 31.3 & $8.6-54.0$ \\
\hline $\begin{array}{l}\text { Median } \\
\text { OS (mos) }\end{array}$ & 24.5 & & $15.7-33.3$ & 24.5 & & $9.1-39.8$ & 22.9 & & $6.7-39.0$ \\
\hline \multicolumn{10}{|l|}{ OS rate } \\
\hline $6 \mathrm{mos}$ & & 100 & & & 100 & & & 100 & \\
\hline
\end{tabular}

\begin{tabular}{|c|c|c|c|c|c|c|c|c|c|}
\hline \multirow[t]{2}{*}{ Outcome } & \multicolumn{3}{|c|}{$\begin{array}{l}\text { PP population } \\
(n=36)\end{array}$} & \multicolumn{3}{|c|}{$\begin{array}{l}\text { Patients with positive } \\
\text { MGMT expression } \\
(\mathrm{n}=20)\end{array}$} & \multicolumn{3}{|c|}{$\begin{array}{l}\text { Patients with negative } \\
\text { MGMT expression } \\
(\mathrm{n}=16)\end{array}$} \\
\hline & No. & $\%$ & $95 \% \mathrm{CI}$ & No. & $\%$ & $95 \% \mathrm{CI}$ & No. & $\%$ & $95 \% \mathrm{CI}$ \\
\hline $12 \mathrm{mos}$ & & 83.3 & $71.1-95.5$ & & 80.0 & $62.6-97.4$ & & 87.5 & $71.2-100$ \\
\hline $18 \mathrm{mos}$ & & 66.1 & $50.4-81.8$ & & 58.7 & $36.9-80.5$ & & 75.0 & $53.8-96.2$ \\
\hline $24 \mathrm{mos}$ & & 51.1 & $34.4-67.8$ & & 53.3 & $40.0-75.6$ & & 47.7 & $22.4-73.0$ \\
\hline
\end{tabular}

*: response of combination therapy at the end of radiotherapy.

Abbreviations: PP: per-protocol; MGMT: O6-methylguanine-DNA

methyltransferase; CR: complete remission; PR : partial remission; SD: stable disease; PD: progressive disease; OS: overall survival; PFS: progression-free survival; $95 \%$ CI: $95 \%$ confidence interval; mos: months.

\section{Safety}

During the RT period, the combination of nimotuzumab with standard TMZ/RT was safe and well tolerated. Treatment-related AEs were usually mild to moderate, self-limiting, reversible, and within the range previously observed with TMZ/RT alone (Table 4) [3]. The most common AEs were constipation, anorexia, fatigue, nausea, vomiting and leucopenia. One patient $(2.6 \%)$ who was a hepatitis B 
virus carrier, experienced TMZ-related grade 3 liver toxicities. Four patients $(10.3 \%)$ presented with mild nimotuzumab-related skin rash. No allergic reaction was reported.

During the adjuvant-therapy period, constipation, anorexia, fatigue, nausea, vomiting and leucopenia were the most common AEs (Table 4). Two patients $(6.1 \%)$ discontinued the treatment because of grade 3 AEs. One experienced severe pneumonia during the first TMZ cycle; another experienced tympanitis related to $\mathrm{RT}$ and refused the TMZ treatment.

A

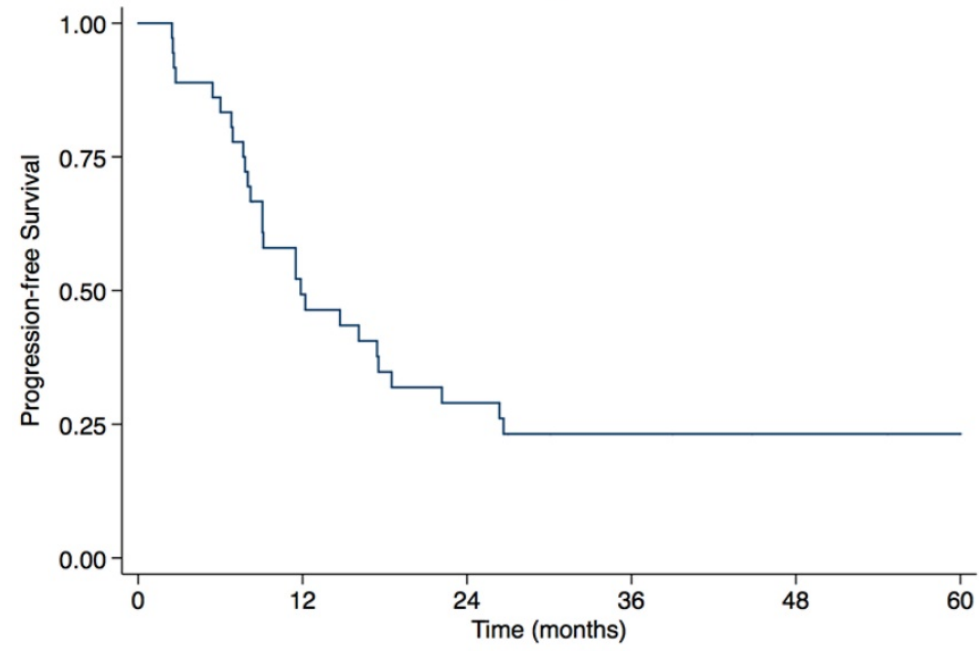

B

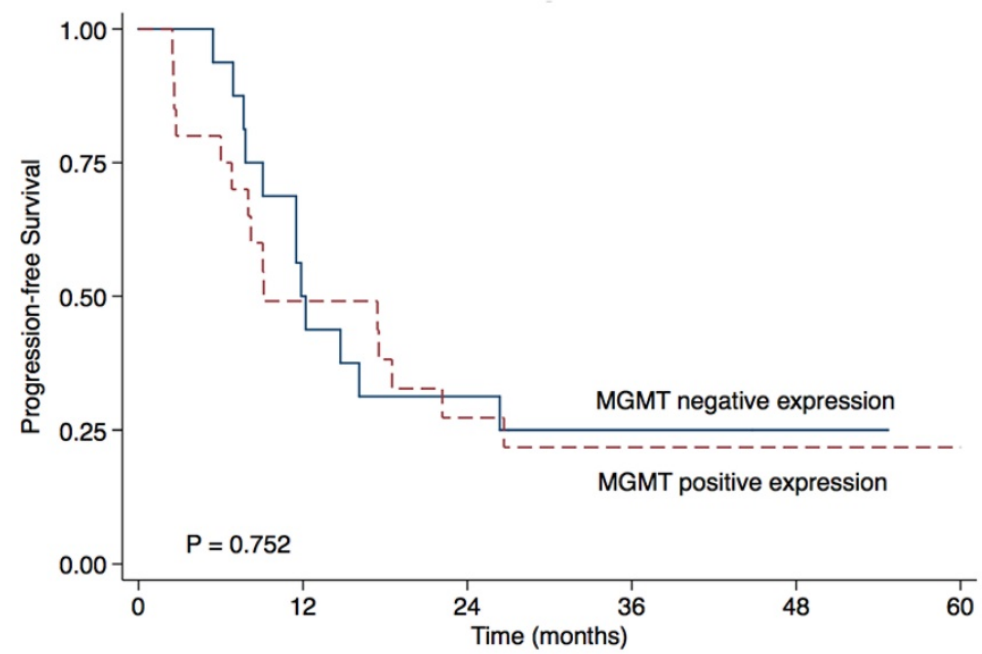

Figure 3. Kaplan-Meier estimate for progression-free survival for (A) all patients and stratified by (B) O6-methylguanine-DNA methyltransferase (MGMT) promoter methylation status.

Table 3. Subgroup survival analysis $(n=36)$

\begin{tabular}{|c|c|c|c|c|c|c|c|c|c|c|c|c|}
\hline \multirow[t]{3}{*}{ Variables } & \multicolumn{6}{|l|}{ PFS } & \multicolumn{6}{|l|}{ OS } \\
\hline & \multicolumn{3}{|c|}{ Univariate analysis } & \multicolumn{3}{|c|}{ Multivariate analysis } & \multicolumn{3}{|c|}{ Univariate analysis } & \multicolumn{3}{|c|}{ Multivariate analysis } \\
\hline & HR & $95 \% \mathrm{CI}$ & $\mathbf{P}$ & HR & 95\%CI & $\mathbf{P}$ & HR & 95\%CI & $\mathbf{P}$ & HR & 95\%CI & $\mathbf{P}$ \\
\hline Age (years): $>40$ vs. $\leq 40$ & 4.45 & $1.32-15.02$ & 0.016 & 4.45 & $1.32-15.02$ & 0.016 & 4.41 & $1.28-15.12$ & 0.018 & 4.41 & $1.28-15.12$ & 0.018 \\
\hline Sex: Male vs. Female & 1.55 & $0.72-3.33$ & 0.260 & & & & 1.35 & $0.61-3.01$ & 0.463 & & & \\
\hline KPS: $60-80$ vs. $90-100$ & 1.30 & $0.58-2.89$ & 0.526 & & & & 2.18 & $0.91-5.20$ & 0.080 & & & \\
\hline Hemisphere: Right vs. Left & 0.66 & $0.30-1.47$ & 0.307 & & & & 0.97 & $0.44-2.18$ & 0.949 & & & \\
\hline Lobe: Others vs. Frontal lobe & 1.11 & $0.47-2.64$ & 0.810 & & & & 1.22 & $0.51-2.94$ & 0.658 & & & \\
\hline Resection: Sub-total vs. Total & 1.95 & $0.88-4.35$ & 0.102 & & & & 1.48 & $0.65-3.37$ & 0.352 & & & \\
\hline MGMT: Positive vs. Negative & 1.13 & $0.53-2.42$ & 0.753 & & & & 1.29 & $0.58-2.89$ & 0.529 & & & \\
\hline Response*: Others vs. CR & 2.69 & $1.25-5.75$ & 0.011 & & & & 2.09 & $0.93-4.73$ & 0.075 & & & \\
\hline
\end{tabular}

*: response of combination therapy at the end of radiotherapy.

Abbreviations: KPS, Karnofsky performance score; MGMT: O6-methylguanine-DNA methyltransferase; OS, overall survival; PFS, progression-free survival; 95\% CI: 95\% confidence interval; HR: hazard ratio; CR: complete remission. 
Table 4. Adverse Events*

\begin{tabular}{|c|c|c|c|c|c|c|c|c|}
\hline \multirow[t]{2}{*}{ Toxicity } & \multicolumn{4}{|c|}{ Radiotherapy period $(n=36)$} & \multicolumn{4}{|c|}{ Adjuvant therapy period $(n=32)$} \\
\hline & All Grades & Grade 1 & Grade 2 & Grade 3 & All Grades & Grade 1 & Grade 2 & Grade 3 \\
\hline & No. $(\%)$ & No. $(\%)$ & No. $(\%)$ & No. (\%) & No. $(\%)$ & No. $(\%)$ & No. $(\%)$ & No. $(\%)$ \\
\hline \multicolumn{9}{|l|}{ Hematologic } \\
\hline Leukopenia & $14(38.9)$ & $10(27.8)$ & $4(11.1)$ & $0(0.0)$ & $14(43.7)$ & $8(25.0)$ & $5(15.6)$ & $1(3.1)$ \\
\hline Neutropenia & $5(13.9)$ & $3(8.3)$ & $2(5.6)$ & $0(0.0)$ & $8(25.0)$ & $3(9.4)$ & $4(12.5)$ & $1(3.1)$ \\
\hline Anemia & $11(30.6)$ & $11(30.6)$ & $0(0.0)$ & $0(0.0)$ & $2(6.2)$ & $2(6.2)$ & $0(0.0)$ & $0(0.0)$ \\
\hline Thrombocytopenia & $3(8.3)$ & $3(8.3)$ & $0(0.0)$ & $0(0.0)$ & $0(0.0)$ & $0(0.0)$ & $0(0.0)$ & $0(0.0)$ \\
\hline \multicolumn{9}{|l|}{ Non-hematologic } \\
\hline Constipation & $21(58.3)$ & $20(55.6)$ & $1(2.8)$ & $0(0.0)$ & $18(56.2)$ & $17(53.1)$ & $1(3.1)$ & $0(0.0)$ \\
\hline Anorexia & $12(33.3)$ & $10(27.8)$ & $2(5.6)$ & $0(0.0)$ & $16(50.0)$ & $14(43.7)$ & $2(6.2)$ & $0(0.0)$ \\
\hline Fatigue & $11(30.6)$ & $10(27.8)$ & $1(2.8)$ & $0(0.0)$ & $16(50.0)$ & $12(37.5)$ & $3(9.4)$ & $1(3.1)$ \\
\hline Nausea & $10(27.8)$ & $8(22.2)$ & $2(5.6)$ & $0(0.0)$ & $9(28.1)$ & $8(25.0)$ & $1(3.1)$ & $0(0.0)$ \\
\hline Vomiting & $7(19.4)$ & $5(13.9)$ & $2(5.6)$ & $0(0.0)$ & $7(21.9)$ & $3(9.4)$ & $4(12.5)$ & $0(0.0)$ \\
\hline Infection & $3(8.3)$ & $1(2.8)$ & $2(5.6)$ & $0(0.0)$ & $1(3.1)$ & $0(0.0)$ & $0(0.0)$ & $1(3.1)$ \\
\hline Diarrhea & $2(5.6)$ & $2(5.6)$ & $0(0.0)$ & $0(0.0)$ & $1(3.1)$ & $0(0.0)$ & $1(3.1)$ & $0(0.0)$ \\
\hline Fever & $1(2.8)$ & $0(0.0)$ & $1(2.8)$ & $0(0.0)$ & $1(3.1)$ & $0(0.0)$ & $0(0.0)$ & $1(3.1)$ \\
\hline ALT elevation & $5(13.9)$ & $4(11.1)$ & $0(0.0)$ & $1(2.8)$ & $1(3.1)$ & $1(3.1)$ & $0(0.0)$ & $0(0.0)$ \\
\hline AST elevation & $1(2.8)$ & $0(0.0)$ & $0(0.0)$ & $1(2.8)$ & $0(0.0)$ & $0(0.0)$ & $0(0.0)$ & $0(0.0)$ \\
\hline Creatinine elevation & $1(2.8)$ & $1(2.8)$ & $0(0.0)$ & $0(0.0)$ & $0(0.0)$ & $0(0.0)$ & $0(0.0)$ & $0(0.0)$ \\
\hline Tympanitis & $0(0.0)$ & $0(0.0)$ & $0(0.0)$ & $0(0.0)$ & $1(3.1)$ & $0(0.0)$ & $0(0.0)$ & $1(3.1)$ \\
\hline Rash & $4(11.1)$ & $3(8.3)$ & $1(2.8)$ & $0(0.0)$ & $0(0.0)$ & $0(0.0)$ & $0(0.0)$ & $0(0.0)$ \\
\hline
\end{tabular}

Abbreviations: ALT, alanine aminotransferase; AST, aspartate aminotransferase. *Adverse events were graded based on National Cancer Institute Common Terminology Criteria for Adverse Events (version 3.0).

\section{Discussion}

GBM is a deadly brain cancer that calls for more effective treatment. The current standard of care includes surgical resection, adjuvant radiotherapy and chemotherapy which have shown limited efficacy. Current standard treatment (TMZ/RT $\rightarrow$ TMZ) was proposed by Stupp $R$ et al. in 2005 and became popularized globally thereafter. Here we show that addition of nimotuzumab successfully prolonged the median PFS by 5.0 months (11.9 months vs. 6.9 months) and median OS by 9.9 months (24.5 months vs. 14.6 months), which translated to a PFS benefit of $18.3 \%$ and an OS benefit of $24.6 \%$ in two years [3].

Overexpression of EGFRvIII in human GBM cells, a functional and permanently activated mutation of the EGFR, enhances the capacity of unregulated growth of tumor, by stimulating cancer cells proliferation, inducing angiogenesis and conferring chemo-resistance in cell and mice [21-23]. Inhibition of EGFRvIII by a tyrosine kinase inhibitor AG1478 restores sensitivity of GBM cells to cisplatin, and suppress growth of GBM xenografts [24]. These results suggest that targeting EGFR signaling could be an effective approach for high-grade glioma therapy.

Nimotuzumab is a monoclonal antibody that selectively blocks EGFR signaling, by binding to an epitope in the extracellular domain III of EGFR that is preserved in the mutant EGFRvIII. It has been reported that the inhibition of miR-566 may sensitize glioblastoma cells to nimotuzumab [25]. Nimotuzumab alone or combined with other chemotherapeutic drugs can suppress cancer proliferation and angiogenesis as well as enhance radio-sensitivity and chemo-sensitivity in tumors that overexpress EGFR in pre-clinical studies [26-29]. The intracavitary administration of nimotuzumab has been explored in recurrent high-grade glioma [30].

Clinical benefit of nimotuzumab combined with RT has been demonstrated in high-grade glioma in several trials, both in adults and pediatric population [31]. Ramos et al. conducted a multicenter Phase I/II trial treating 29 newly diagnosed high-grade glioma patients (GBM, 16 cases) with 6 weekly infusions of nimotuzumab in $200 \mathrm{mg}$ doses in combination with RT [12]. Nimotuzumab was well tolerated and the median OS reached 17.47 months for GBM. Solomon et al. did a single institution study with nimotuzumab plus RT in treating high-grade glioma and showed a median OS of 12.4 months for GBM, which was longer than the 8.0 months in a matched population with RT alone at the same hospital [13]. The efficacy of nimotuzumab plus RT was tested in a randomized multicenter Phase III clinical study in 70 high-grade glioma patients (GBM, 29 cases). Nimotuzumab showed significant survival benefit with a 5-month prolongation in median OS than with RT alone (17.76 vs. 12.63 months) [14].

However, adding nimotuzumab to chemo-RT has not resulted in satisfactory outcomes so far. In 2012, Hong et al. tested the therapeutic effects of nimotuzumab in addition to the standard TMZ/RT $\rightarrow$ TMZ therapy in 41 malignant glioma patients. Although there was a trend towards survival benefit with the addition of nimotuzumab (median OS, 16.5 vs. 10.5 months), no significant difference was found in this Phase I/II trial [15]. Wang et al evaluated the efficacy of a combination of nimotuzumab and $\mathrm{TMZ} / \mathrm{RT} \rightarrow \mathrm{TMZ}$ in 26 newly diagnosed GBM [16]. 
The median PFS of patients treated with nimotuzumab plus TMZ/RT $\rightarrow \mathrm{TMZ}$ was slightly longer than that of patients treated with standard therapy (10.0 vs. 6.9 months), but the median OS did not show a survival advantage (15.9 vs 14.6 months). Westphal et al. conducted a Phase III trial in which 149 GBM patients were randomly assigned to receive either nimotuzumab (400 mg weekly in followed by $400 \mathrm{mg}$ biweekly after 12 weeks) combined with standard TMZ/RT $\rightarrow$ TMZ or standard TMZ/RT $\rightarrow$ TMZ alone [17]. The median OS between patients in nimotuzumab group and standard group was not significantly different (22.3 vs. 19.6 months). It is noted that the median OS of 19.6 months in the standard care arm exceeds the previous reported 14.6 months in the study by Stupp et al. [3].

Our study is the first Phase II trial to evaluate the clinical effects of nimotuzumab plus standard therapy in GBM patients all with positive EGFR expression. The survival time in present study exceeds all those previously reported. In the study by Wang et al. [16], $50.0 \%(13 / 26)$ patients were positive EGFR expression and in the study of Westphal et al. [17] $28.2 \%(20 / 71)$ were reported with EGFR amplification. EGFR expression has been associated with aggressiveness and poor survival in malignant gliomas. It has been reported that the survival in EGFR expressing GBM patients was significantly less than that in non-expressing patients receiving conventional therapy (median OS, 12.5 vs. 17.5 months, $P=0.013$ ) [32]. Therefore, it is reasonable that nimotuzumab was a vital intervention, contributing to the survival in this study. We speculate that variation in the EGFR expression among the recruited patients in previous studies led the less favorable effect. It has been reported that proximately 10\% GBM patients over-expressed EGFRvIII [33]. Unfortunately, we were unable to distinguish the wild-type EGFR from EGFRvIII in fixed tumour specimens due to limitation in our method.

No significant correlation between treatment outcomes and MGMT status was found, which suggests an efficacy of adding nimotuzumab to dose-dense TMZ in GBM patients with MGMT positive expressing. The median OS for our patients with positive MGMT expression reached 24.5 months, comparable with that of patients with negative MGMT expression in the same study, which is much longer than the reported 12.6 months with standard therapy [34]. We used an intensive adjuvant TMZ regimen of a 7-days on/7-days off in patients with positive MGMT expression. MGMT plays a vital role in regulating TMZ sensitivity. The negative expression of the methylated MGMT gene promoter in tumor tissues enables GBM patients responding to standard TMZ/RT therapy. Positive MGMT expression might eliminate such benefit [35]. Dose-intense schedules of TMZ have been tested for GBM patients recently. However, one dose-dense TMZ schedule (21-days on/7-days off after completion of combined TMZ/RT in newly-diagnosed GBM) failed to demonstrate survival benefit regardless of MGMT status in a large phase III trial (RTOG 0525) [36]. Nevertheless, Westphal et al. reported a favorable trend in patients with positive MGMT expression with the addition of nimotuzumab [17].

The percentage of total resection $(41.7 \%)$ in present study is similar to that of Westphal et al. $(43.7 \%)$ [17], and higher than that of Hong et al. $(20 \%)$ and Wang et al. $(34.6 \%)[15,16]$. The effect of surgical resection extent in the treatment of GBM is still under debate [37-39]. In the present study, no correlation was found between surgical resection extent and clinical outcomes.

Treatment-related AEs were mild to moderate. Most of the reported AEs are previously known to the primary disease process or the TMZ/RT alone.

\section{Conclusions}

In this multicenter Phase II trial, we show that nimotuzumab in combination with standard $\mathrm{TMZ} / \mathrm{RT} \rightarrow \mathrm{TMZ}$ has an excellent safety profile and benefit in increase of survival in patients with newly diagnosed GBM. Furthermore, the addition of nimotuzumab may provide benefits especially for patients with positive MGMT expression. This encouraging result calls for randomized, double blind, Phase III clinical trials.

\section{Abbreviations}

EGFR: epidermal growth factor receptor.

EGFRvIII: EGFR variant III mutant.

GBM: glioblastoma multiforme.

PFS: progression-free survival.

OS: overall survival.

ORR: objective response rate.

MGMT: O (6)-methylquanine DNA methyltranferase.

RT: radiotherapy.

TMZ: temozolomide.

KPS: Karnofsky performance score.

ULN: upper limit of normal.

GTV: gross tumor volume.

CTV: clinical target volume.

PTV: planning tumor volume.

MRI: magnetic resonance imaging.

AEs: adverse events.

CR: complete remission.

PR: partial remission. 
SD: stable disease.

PD: progressive disease.

PP: per-protocol.

CI: confidence interval.

HR: hazard ratio.

ALT: alanine aminotransferase.

AST: aspartate aminotransferase.

\section{Acknowledgements}

We appreciate the anonymous reviewer for their insightful comments and great efforts to improve this manuscript.

\section{Ethics approval and consent to participate}

The study was approved by the Institutional Review Board of Sun Yat-sen University Cancer Center, and was conducted in accordance with the Good Clinical Practice guideline. The ethics approval was for all the study sites. The investigator obtained signed informed consent form from patients for approval of treatment and for personal information use in research without violating their privacy. The study was performed in accordance with the Declaration of Helsinki.

\section{Consent for publication}

Written informed consent for publication has been obtained.

\section{Funding}

This study was sponsored by Biotech Pharmaceutical Co., Ltd. The funding body didn't participate in any period of the entire research process, including the design of the study, collection, analysis, interpretation of data and writing the manuscript.

\section{Authors' Contributions}

Conception and design: SXW.

Development of methodology: XML, LBC, JCS, SYW, XCW, PP, ZPC, SXW.

Acquisition of data (provided animals, acquired and managed patients, provided facilities, etc.): XJD, XML, LBC, JCS, SYW, XCW, XLP, MLD, FFC, ZQW, FRC, HHZ, HYW.

Analysis and interpretation of data (e.g., statistical analysis, biostatistics, computational analysis): XJD, FFC, ZQW, HHZ, SXW.

Writing, review, and/or revision of the manuscript: XJD, PP, JL, SXW.

Administrative, technical, or material support (i.e., reporting or organizing data, constructing databases): XJD, SXW.

Study supervision: ZPC, JL, SXW.
Clinical Trial Registration

NCT03388372.

\section{Competing Interests}

The authors have declared that no competing interest exists.

\section{References}

1. Wen PY, Kesari S. Malignant gliomas in adults. N Engl J Med. 2008; 359(5):492-507.

2. Affronti ML, Heery CR, Herndon JE 2nd, et al. Overall survival of newly diagnosed glioblastoma patients receiving carmustine wafers followed by radiation and concurrent temozolomide plus rotational multiagent chemotherapy. Cancer. 2009;115(15):3501-3511.

3. Stupp R, Mason WP, Van Den Bent MJ, et al. Radiotherapy plus concomitant and adjuvant temozolomide for glioblastoma. N Engl J Med. 2005;352(10):987-96.

4. Arteaga CL. The epidermal growth factor receptor: From mutant oncogene in nonhuman cancers to therapeutic target in human neoplasia. J Clin Oncol. 2001;19(Suppl 18):S32-S40.

5. Hoi Sang U, Espiritu OD, Kelley PY, et al. The role of the epidermal growth factor receptor in human gliomas: Ii. The control of glial process extension and the expression of glial fibrillary acidic protein. J Neurosurg. 1995; 82(5):847-857

6. Schlegel J, Merdes A, Stumm G, et al. Amplification of the epidermal-growth-factor-receptor gene correlates with different growth behaviour in human glioblastoma. Int J Cancer. 1994;56(1):72-7.

7. Diaz Miqueli A, Blanco R, Garcia B, et al. Biological activity in vitro of anti-epidermal growth factor receptor monoclonal antibodies with different affinities. Hybridoma. 2007; 26(6):423-431.

8. Stockhausen MT, Broholm H, Villingshoj M, et al. Maintenance of EGFR and EGFRvIII expressions in an in vivo and in vitro model of human glioblastoma multiforme. Exp Cell Res. 2011;317(11):1513-26.

9. Viana-Pereira M, Lopes JM, Little S, et al. Analysis of EGFR overexpression, EGFR gene amplifcation and the EGFRvIII mutation in Portuguese high-grade gliomas. Anticancer Res. 2008;28:913-920.

10. Crombet T, Osorio $\mathrm{M}, \mathrm{Cruz} \mathrm{T}$, et al. Use of the humanized anti-epidermal growth factor receptor monoclonal antibody h-R3 in combination with radiotherapy in the treatment of locally advanced head and neck cancer patients. J Clin Oncol. 2004 22(9):1646-54.

11. Reddy BK, Lokesh V, Vidyasagar MS, et al. Nimotuzumab provides survival benefit to patients with inoperable advanced squamous cell carcinoma of the head and neck: A randomized, open-label, phase IIb, 5-year study in indian patients. Oral Oncol. 2014;50(5):498-505.

12. Ramos TC, Figueredo J, Catala M, et al. Treatment of high-grade glioma patients with the humanized anti-epidermal growth factor receptor (EGFR) antibody h-R3: report from a phase I/II trial. Cancer Biol Ther. 2006:5(4):375-9.

13. Solomon MT, Miranda N, Jorrín E, et al. Nimotuzumab in combination with radiotherapy in high grade glioma patients: a single institution experience. Cancer Biol Ther. 2014;15(5):504-509.

14. Solomon MT, Selva JC, Figueredo J, et al. Radiotherapy plus nimotuzumab or placebo in the treatment of high grade glioma patients: Results from a randomized, double blind trial. BMC Cancer. 2013:13.299.

15. Hong J, Peng Y, Liao $Y$, et al. Nimotuzumab prolongs survival in patients with malignant gliomas: A phase I/II clinical study of concomitant radiochemotherapy with or without nimotuzumab. Exp Ther Med. 2012;4(1):151-157.

16. Wang Y, Pan L, Sheng XF, et al. Nimotuzumab, a humanized monoclonal antibody specific for the EGFR, in combination with temozolomide and radiation therapy for newly diagnosed glioblastoma multiforme: first results in Chinese patients. Asia Pac J Clin Oncol. 2016;12(1):e23-9.

17. Westphal M, Heese O, Steinbach JP, et al. A randomised, open label phase III trial with nimotuzumab, an anti-epidermal growth factor receptor monoclonal antibody in the treatment of newly diagnosed adult glioblastoma. Eur J Cancer. 2015;51(4):522-532.

18. Wen PY, Macdonald DR, Reardon DA, et al. Updated response assessment criteria for high-grade gliomas: response assessment in neuro-oncology working group. J Clin Oncol. 2010; 28(11):1963-72.

19. Combs S, Schulz-Ertner D, Hartmann C, et al. Phase I/II study of cetuximab plus temozolomide as radiochemotherapy for primary glioblastoma (GERT)--eudract number 2005-003911-63. NCT00311857. ASCO Annual Meeting Proceedings, 2008:2077.

20. Yoon Ks, Lee MC, Kang SS, et al. p53 mutation and epidermal growth factor receptor overexpression in glioblastoma. J Korean Med Sci. 2001:16(4):481-8.

21. Nagane, M, Coufal F, Lin H, et al. A common mutant epidermal growth factor receptor confers enhanced tumorigenicity on human glioblastoma cells by increasing proliferation and reducing apoptosis. Cancer Res. 1996; 56:5079-5086. 
22. Bonavia $\mathrm{R}$, Inda $\mathrm{MM}$, Vandenberg $\mathrm{S}$, et al. EGFRvIII promotes glioma angiogenesis and growth through the NF-kappaB, interleukin-8 pathway. Oncogene. 2012; 31:4054-4066.

23. Nagane M, Levitzki A, Gazit A, et al. Drug resistance of human glioblastoma cells conferred by a tumor-specific mutant epidermal growth factor receptor through modulation of Bcl-XL and caspase-3- like proteases. Proc Natl Acad Sci U S A. 1998:95:5724-5729.

24. Nagane M, Narita Y, Mishima K, et al. Human glioblastoma xenografts overexpressing a tumor-specific mutant epidermal growth factor receptor sensitized to cisplatin by the AG1478 tyrosine kinase inhibitor. J Neurosurg. 2001; 95:472-479.

25. Zhang KL, Zhou X, Han L, Chen LY, Chen LC, Shi ZD, et al. MicroRNA-566 activates EGFR signaling and its inhibition sensitizes glioblastoma cells to nimotuzumab. Mol Cancer. 2014; 13: 63.

26. Crombet-Ramos T, Rak J, Pérez R, Viloria-Petit A. Antiproliferative, antiangiogenic and proapoptotic activity of h-R3: A humanized anti-EGFR antibody. Int J Cancer. 2002;101(6):567-75.

27. Diaz Miqueli A, Rolff J, Lemm M, et al. Radiosensitisation of U87MG brain tumours by anti-epidermal growth factor receptor monoclonal antibodies. Br J Cancer. 2009;100(6):950-8.

28. Nitta Y, Shimizu S, Shishido-Hara Y, et al. Nimotuzumab enhances temozolomide-induced growth suppression of glioma cells expressing mutant EGFR in vivo. Cancer Med. 2016;5(3):486-99.

29. Chong DQ Toh XY, Ho IA, et al. Combined treatment of Nimotuzumab and rapamycin is effective against temozolomide-resistant human gliomas regardless of the EGFR mutation status. BMC Cancer. 2015; 15:255.

30. Casacó A, López G, García I, et al. Phase I single-dose study of intracavitary-administered Nimotuzumab labeled with 188 Re in adult recurrent high-grade glioma. Cancer Biol Ther. 2008;7(3):333-9.

31. Yang QY, Guo CC, Chen ZP. Profile of nimotuzumab in the treatment of high-grade glioma. Onco Targets Ther. 2015; 8:819-25.

32. Choi Y, Song YJ, Lee HS, et al. Epidermal growth factor receptor is related to poor survival in glioblastomas: single-institution experience. Yonsei Med J. 2013;54(1):101-7.

33. Das A, Tan WL, Teo J, Smith DR. Glioblastoma multiforme in an Asian population: evidence for a distinct genetic pathway. J Neurooncol. 2002;60(2):117-25.

34. Watanabe $\mathrm{R}$, Nakasu $\mathrm{Y}$, Tashiro $\mathrm{H}$, et al. O6-methylguanine DNA methyltransferase expression in tumor cells predicts outcome of radiotherapy plus concomitant and adjuvant temozolomide therapy in patients with primary glioblastoma. Brain Tumor Pathol. 2011; 28(2):127-35.

35. Hegi ME, Diserens AC, Gorlia T, et al. MGMT gene silencing and benefit from temozolomide in glioblastoma. N Engl J Med. 2005;352(10):997-1003.

36. Gilbert MR, Wang M, Aldape KD, et al. Dose-dense temozolomide for newly diagnosed glioblastoma: a randomized phase III clinical trial. J Clin Oncol. 2013;31(32):4085-91.

37. Simpson JR, Horton J, Scott C, et al. Influence of location and extent of surgical resection on survival of patients with glioblastoma multiforme: results of three consecutive Radiation Therapy Oncology Group (RTOG) clinical trials. Int J Radiat Oncol Biol Phys. 1993;26:239-244

38. Kowalczuk A, Macdonald RL, Amidei C, et al. Quantitative imaging study of extent of surgical resection and prognosis of malignant astrocytomas. Neurosurgery. 1997;41(5):1028-36.

39. Lacroix M, Abi-Said D, Fourney DR, et al. A multivariate analysis of 416 patients with glioblastoma multiforme: prognosis, extent of resection, and survival. J Neurosurg. 2001;95(2):190-8 